Europhysics Letters

PREPRINT

\title{
Quantum state preparation in circuit QED via Landau- Zener tunneling
}

\author{
Keiji Saito ${ }^{1}$, Martijn Wubs ${ }^{2}$, Sigmund Kohler ${ }^{2}$, Peter HängGi ${ }^{2}$ and Yosuke \\ KAYANUMA $^{3}$ \\ 1 Department of Physics, Graduate School of Science, University of Tokyo - Tokyo 113- \\ 0033, Japan \\ 2 Institut für Physik, Universität Augsburg - D-86135 Augsburg, Germany \\ 3 Department of Mathematical Science, Graduate School of Engineering, Osaka Prefec- \\ ture University - Sakai 599-8531, Japan
}

PACS. 32.80.Qk - Coherent control of photon-atom interactions.

PACS. 03.67.Lx - Quantum computation.

PACS. 32.80.Bx - Level crossing and optical pumping.

\begin{abstract}
We study a qubit undergoing Landau-Zener transitions enabled by the coupling to a circuit-QED mode. Summing an infinite-order perturbation series, we determine the exact nonadiabatic transition probability for the qubit, being independent of the frequency of the QED mode. Possible applications are single-photon generation and the controllable creation of qubit-oscillator entanglement.
\end{abstract}

Superconducting loops are promising candidates for solid state qubit implementations [1-4]. Since the direct observation of Rabi-oscillations in these systems [1], they form the basis of many experiments on coherent quantum dynamics. Particularly interesting are the experiments in circuit quantum electrodynamics (QED) [2-4], which is the solid-state analogue of a two-level atom in an optical cavity. Superconducting circuits possess the advantage that many of their parameters are tunable over a broad range. This can be exploited for controlling efficiently the qubits.

One particular way of controlling a qubit works by switching the difference of its diabatic energies from a large negative to a large positive value, yielding an avoided crossing for the adiabatic energies. For sufficiently slow switching, the qubit will adiabatically follow its instantaneous eigenstates. In the opposite limit of fast switching, however, the qubit will abandon the adiabatic eigenstate and undergo a so-called Landau-Zener (LZ) transition. The LZ transition probability can be raised upon increasing the switching rate [5-7]. LZ transitions can be used to effectively control qubit gate operations [8,9] and to read out qubits [10]. Recently, LZ transitions have been observed in various experiments with superconducting qubits [11-14] and nanomagnets [15].

Landau-Zener transitions can also occur for a qubit that is coupled to a circuit oscillator. Then, the adiabatic following to the final ground state takes place even in the absence of a direct coupling between the two qubit levels, induced instead by the indirect coupling to the

(C) EDP Sciences 
oscillator. An oscillator in a highly excited coherent state can be described classically, yielding a non-monotonic behavior of the LZ transition probability versus the coupling strength [16].

Experimentally relevant is also the opposite limit in which the oscillator is initially in its ground state. Here, we shall investigate the Landau-Zener dynamics of such a qubit-oscillator setup and make specific suggestions for exploiting it to manipulate the quantum states of the qubit and the oscillator. We derive an exact analytical expression for the transition probabilities and we propose the creation of single circuit photons.

In tunneling representation, a circuit QED setup is described by the Hamiltonian $[2,3]$

$$
H(t)=-\frac{E_{\mathrm{el}}}{2} \sigma_{x}-\frac{E_{\mathrm{J}}(t)}{2} \sigma_{z}+\hbar \Omega b^{\dagger} b+\gamma\left(b^{\dagger}+b\right)\left[\sigma_{x}+1-2 N_{\mathrm{g}}\right]
$$

for the qubit, the circuit oscillator, and their mutual coupling. The cavity is modelled as a harmonic oscillator with the annihilation and creation operators $b$ and $b^{\dagger}$, while $\sigma_{x}$ and $\sigma_{z}$ denote Pauli matrices. The electrostatic energy $E_{\mathrm{el}}=4 E_{\mathrm{c}}\left[1-2 N_{\mathrm{g}}\right]$ is determined by the charging energy $E_{\mathrm{c}}$ and the tunable gate charge $N_{\mathrm{g}}$. The tunable flux $\Phi(t)$ penetrating the superconducting loop will be used to drive the qubit. The flux controls the Josephson energy $E_{\mathrm{J}}(t)=E_{\mathrm{J}, \max } \cos \left[\pi \Phi(t) / \Phi_{0}\right]$, where $\Phi_{0}$ is the flux quantum. The two-level approximation underlying the Hamiltonian (11) is valid in the charge regime $E_{\mathrm{c}} \gg E_{\mathrm{J}}$. In order to minimize decoherence, one typically operates the qubit at the optimal working point $N_{\mathrm{g}}=\frac{1}{2}$, so that $E_{\mathrm{el}}=0$ [17]. Henceforth, we restrict ourselves to this optimal working point. The LZ dynamics can then be realised by switching the flux $\Phi(t)$ in such a way that $E_{\mathrm{J}}(t)=-v t$, with $v>0$. The duration of this linear sweep has to be long enough, so that transition probabilities have converged and the finite time interval can be extended to $t=-\infty \ldots \infty$ in calculations describing the dynamics. Since the energy splitting of the qubit can even vanish, it is essential to treat the qubit-oscillator coupling beyond rotating-wave approximation. The temperatures in circuit QED experiments [2] justify the assumption that both the qubit and the oscillator are initially in their ground states, i.e. $|\Psi(-\infty)\rangle=|\uparrow, 0\rangle$, where $\sigma_{z}|\uparrow\rangle=|\uparrow\rangle$.

Nonadiabatic transitions. - For $E_{\mathrm{el}}=0$, the states $|\uparrow\rangle$ and $|\downarrow\rangle$ are eigenstates of the qubit Hamiltonian $\frac{1}{2} v t \sigma_{z}$ and any transition between these states can only result from the coupling to the oscillator. The central quantity of interest is the probability $P_{\uparrow \rightarrow \downarrow}(t)=1-P_{\uparrow \rightarrow \uparrow}(t)$ that the qubit has flipped to the state $|\downarrow\rangle$. In the following, we derive an exact expression for $P_{\uparrow \rightarrow \uparrow}(\infty)=\sum_{n}|\langle\uparrow, n|U(\infty,-\infty)| \uparrow, 0\rangle|^{2}$ where $U\left(t, t^{\prime}\right)$ denotes the time-evolution operator and $|n\rangle$ an oscillator eigenstate. We start by a transformation to an interaction picture with respect to the uncoupled qubit and oscillator, $U_{0}(t)=\exp \left(-\mathrm{i} \Omega b^{\dagger} b t\right) \exp \left(-\frac{\mathrm{i}}{4 \hbar} v t^{2} \sigma_{z}\right)$. This yields the interaction-picture Hamiltonian

$$
\tilde{H}(t)=\gamma\left(b^{\dagger} e^{\mathrm{i} \Omega t}+b e^{-\mathrm{i} \Omega t}\right) \exp \left(-\frac{\mathrm{i}}{2 \hbar} v t^{2} \sigma_{z}\right) \sigma_{x}
$$

A perturbation expansion of the probability amplitude $A_{n}=\langle\uparrow, n|U(\infty,-\infty)| \uparrow, 0\rangle$ results in the series $A_{n}=\sum_{k=0}^{\infty}(-\mathrm{i} / \hbar)^{2 k} a_{n k}$, with the $2 k$-th order contribution $a_{n k}$ equal to

$$
\sum_{\lambda_{2 k} \cdots \lambda_{1}} \gamma^{2 k} C_{n k}(\{\lambda\}) \int_{-\infty}^{\infty} \mathrm{d} t_{2 k} \int_{-\infty}^{t_{2 k}} \mathrm{~d} t_{2 k-1} \ldots \int_{-\infty}^{t_{2}} \mathrm{~d} t_{1} \exp \left[\mathrm{i} \sum_{\ell=1}^{2 k}\left(\Omega \lambda_{\ell} t_{\ell}+\frac{v}{2 \hbar}\left(t_{2 \ell}^{2}-t_{2 \ell-1}^{2}\right)\right)\right] .
$$

Since each $\tilde{H}(t)$ flips the qubit exactly once, only even orders of $\gamma$ appear in $A_{n}$. The coefficients $\lambda_{\ell}= \pm 1$, for $\ell=1, \ldots, 2 k$, stem from the sign in the time-dependent phase of the creation and annihilation operators; $C_{n k}(\{\lambda\})=C_{n k}\left(\lambda_{2 k}, \ldots, \lambda_{1}\right)=\langle n|\cdots| 0\rangle$, where the dots 
denote the combination of $2 k$ creation and annihilation operators $b$ and $b^{\dagger}$ that corresponds to the sequence $\lambda_{2 k}, \ldots, \lambda_{1}$. An important simplification of the $\lambda$-summation results from the fact that $C_{n k}=0$ whenever more annihilation than creation operators act on the oscillator ground state $|0\rangle$. Thus, we need to consider only those $\lambda$-sequences that fulfill the relation

$$
\sum_{\ell^{\prime}=1}^{\ell} \lambda_{\ell^{\prime}} \geq 0, \quad \forall \ell \leq 2 k
$$

For the further evaluation, we substitute in Eq. (3) the times $t_{\ell}$ by the time differences $\tau_{\ell}=t_{\ell+1}-t_{\ell}$, for $\ell=1, \ldots, 2 k-1$, where $t=t_{2 k}$. Thus, we insert $t_{\ell}=t-\sum_{\ell^{\prime}=\ell}^{2 k-1} \tau_{\ell^{\prime}}$, so that the integral in (3) becomes

$$
\int_{-\infty}^{\infty} \mathrm{d} t \int_{0}^{\infty} \mathrm{d} \tau_{2 k-1} \ldots \mathrm{d} \tau_{1} \exp \left[\mathrm{i} \Omega \sum_{\ell=1}^{2 k} \lambda_{\ell}\left(t-\sum_{\ell^{\prime}=\ell}^{2 k-1} \tau_{\ell^{\prime}}\right)+\frac{\mathrm{i} v}{2 \hbar} \sum_{\ell=1}^{k}\left\{2 \tau_{2 \ell-1}\left(t-\sum_{\ell^{\prime}=2 \ell}^{2 k-1} \tau_{\ell^{\prime}}\right)-\tau_{2 \ell-1}^{2}\right\}\right] .
$$

The $t$-integration results in the delta function

$$
2 \pi \delta\left(\frac{v}{\hbar} \sum_{\ell=1}^{k} \tau_{2 \ell-1}+\Omega \sum_{\ell=1}^{2 k} \lambda_{\ell}\right)
$$

From the inequality (4) it follows that the second sum in the argument of the delta function is non-negative. Because the integration interval of all $\tau_{\ell}$ is $[0 \ldots \infty)$, any non-zero contribution to the integral (5) comes from $\tau_{1}=\tau_{3}=\ldots=\tau_{2 k-1}=0$. Hence, the integral over the time differences $\tau_{2}, \tau_{4}, \ldots, \tau_{2 k-2}$ must yield a distribution proportional to $\delta\left(\tau_{1}\right) \delta\left(\tau_{3}\right) \cdots \delta\left(\tau_{2 k-1}\right)$. Evaluating the integrals over all $\tau_{2 \ell}$ separately, one finds that such a distribution is obtained only if $\sum_{\ell^{\prime}=1}^{2 \ell} \lambda_{\ell^{\prime}}=0$ for all $\ell=1, \ldots, k-1$. These $k-1$ relations together with the delta function (16) lead to the conditions $\lambda_{2 \ell}+\lambda_{2 \ell-1}=0$ for all $\ell=1, \ldots, k$. In combination with Eq. (44) they imply that only those integrals with $\lambda_{\ell}=-(-1)^{\ell}$ are non-vanishing. In other words, we obtain the selection rule that to the final occupation probability only those processes contribute in which the oscillator jumps (repeatedly) from the state $|0\rangle$ to the state $|1\rangle$ and back. Hence the only relevant $C_{n k}$ reads $C_{n k}(-1,1, \ldots,-1,1)=\left\langle n\left|\left(b b^{\dagger}\right)^{k}\right| 0\right\rangle=\delta_{n, 0}$.

The remaining multiple integrations are performed as detailed in Refs. $[18,19]$ and result in $a_{n k}=\delta_{n, 0}(\pi \hbar / v)^{k} / k$ !, so that $A_{n}=\delta_{n, 0} \exp \left(-\pi \gamma^{2} / \hbar v\right)$. This implies that the oscillator returns to its ground state provided the qubit ends up in state $|\uparrow\rangle$. Thereby, we arrive at a first central result, namely the exact transition probability

$$
P_{\uparrow \rightarrow \downarrow}(\infty)=1-P_{\uparrow \rightarrow \uparrow}(\infty)=1-\mathrm{e}^{-2 \pi \gamma^{2} / \hbar v} .
$$

Most surprisingly, it does not involve the oscillator frequency $\Omega$. Because of this, Eq. (7) allows a simple interpretation: if the Josephson energy is switched slowly (and the condition for that is $\hbar v \ll \gamma^{2}$ ), then the qubit will follow the adiabatic ground state which at large times is the state $|\downarrow\rangle$. For large $\hbar v / \gamma^{2}$, the qubit will remain in state $|\uparrow\rangle$, corresponding to a nonadiabatic transition. Notice the important difference to the standard LZ problem that an intrinsic coupling between the qubit levels is replaced here by the qubit-oscillator coupling $\gamma$.

Since only the states $|\uparrow, 0\rangle$ and $|\downarrow, 1\rangle$ contribute to the perturbation series for $P_{\uparrow \rightarrow \uparrow}(\infty)$, it is tempting to conclude a posteriori that for the qubit-oscillator coupling, a rotating-wave approximation (RWA) is justified. This would restrict the whole dynamics to the two mentioned states and indeed the same probability $P_{\uparrow \rightarrow \uparrow}(\infty)$ would be obtained. However, below we will 


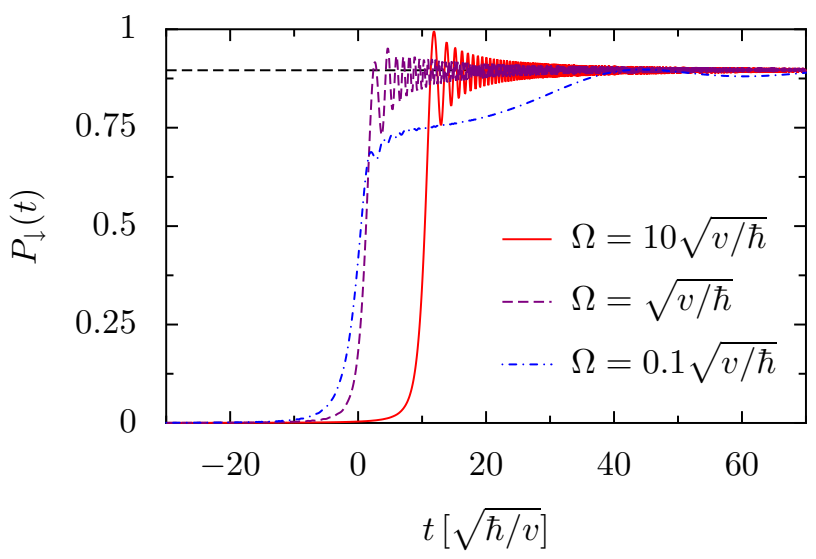

Fig. 1 - Landau-Zener dynamics for the coupling strength $\gamma=0.6 \sqrt{\hbar v}$ for various cavity frequencies $\Omega$. The dashed line marks the $\Omega$-independent, final probability (7) to which all curves converge.

find that at intermediate times, states $|\uparrow, 2 n\rangle$ and $|\downarrow, 2 n+1\rangle$ with $n>0$ can be considerably populated - the latter even at $t \rightarrow \infty$. This demonstrates the surprising fact that the RWA yields the correct transition probability (7) even when the RWA is not justified.

In order to gain information about the dynamics at intermediate times, we numerically integrated the Schrödinger equation for the Hamiltonian (2). The time evolution of the probability that the qubit is in state $|\downarrow\rangle$ is depicted in Fig. 1 It demonstrates that at intermediate times, the dynamics depends strongly on the oscillator frequency $\Omega$, despite the fact that this is not the case for long times. For a large oscillator frequency, $P_{\uparrow \rightarrow \downarrow}(t)$ resembles the standard LZ transition with a time shift $\hbar \Omega / v$.

If the system starts out not in the ground state but in a state $|\uparrow, n\rangle$ with $n>0$, some transition probabilities can still be obtained analytically. We find $P_{\uparrow n \rightarrow \uparrow(n+m)}=0$ for all $m>0$, being a special case of the selection rule derived in Refs. [20,21]. Moreover, $P_{\uparrow n \rightarrow \uparrow n}=$ $\exp \left[-2 \pi(2 n+1) \gamma^{2} / \hbar v\right]$, indicating that $|\uparrow, 0\rangle$ is the most stable state.

Manipulation of the oscillator state. - Having studied the qubit dynamics, we next focus on the modification of the oscillator state. Since we start out in the ground state $|\uparrow, 0\rangle$ and the Hamiltonian (2) correlates every creation or annihilation of a photon with a qubit flip, the resulting dynamics is restricted to the states $|\uparrow, 2 n\rangle$ and $|\downarrow, 2 n+1\rangle$. Figure 2 reveals that the latter states survive for long times, while of the former states only $|\uparrow, 0\rangle$ stays occupied, as it follows from the relation that $A_{n} \propto \delta_{n, 0}$, derived above. Thus, the final state exhibits a peculiar type of entanglement between the qubit and the oscillator, and can be written as

$$
|\Psi(\infty)\rangle=\sqrt{1-P_{\uparrow \rightarrow \downarrow}(\infty)}|\uparrow, 0\rangle+\sqrt{P_{\uparrow \rightarrow \downarrow}(\infty)}\left(c_{1}|\downarrow, 1\rangle+c_{3}|\downarrow, 3\rangle+\ldots\right),
$$

where $\left|c_{1}\right|^{2}+\left|c_{3}\right|^{2}+\ldots=1$. This implies that only odd-photon states are occupied if the qubit ends in $|\downarrow\rangle$, representing a highly nonclassical oscillator state. Qubit and oscillator end up fully entangled, in the sense that after tracing out the oscillator states, no coherence between the qubit states $|\uparrow\rangle$ and $|\downarrow\rangle$ is left.

While $P_{\uparrow \rightarrow \downarrow}(\infty)$ is determined by the ratio $\gamma^{2} / \hbar v$, the coefficients $c_{2 n+1}$ depend also on the oscillator frequency. Experiments in circuit QED have been performed for $\gamma \ll \hbar \Omega$ in the coherent regime [2]. There, $c_{1} \approx 1$ to a very good approximation. Hence one can control via $v$ the final state to be any superposition of $|\uparrow, 0\rangle$ and $|\downarrow, 1\rangle$. In particular, in the adiabatic 


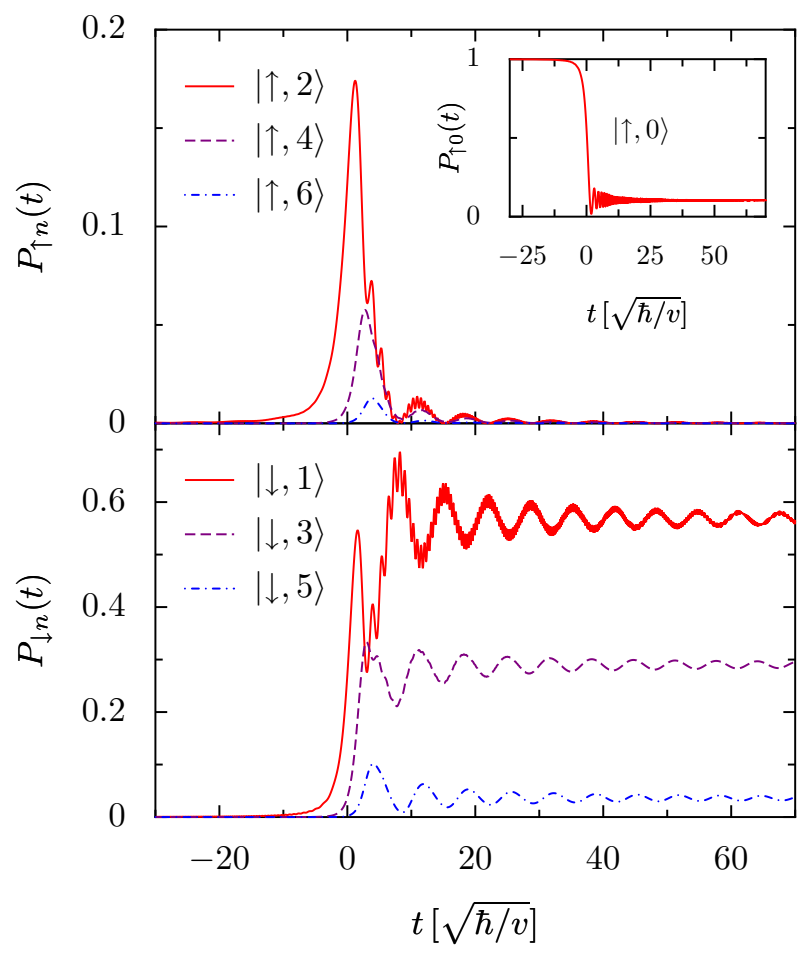

Fig. 2 - Population dynamics of individual qubit-oscillator states for a coupling strength $\gamma=0.6 \sqrt{\hbar v}$ and oscillator frequency $\Omega=0.5 \sqrt{v / \hbar}$.

limit $v \hbar / \gamma^{2} \ll 1$, the final state becomes $|\downarrow, 1\rangle$. This has the important physical implication of the creation of exactly one photon in the cavity, triggered by a Landau-Zener transition. In an experiment, the photon will subsequently leak out of the cavity. By exploiting these two processes, we propose the following four-step LZ cycle for single-photon generation: The first step is single-photon generation in the cavity via the adiabatic LZ transition $|\uparrow, 0\rangle \rightarrow|\downarrow, 1\rangle$, brought about by switching the Josephson energy sufficiently slowly. Second, the photon is released from the cavity via the (controlled) cavity decay $|\downarrow, 1\rangle \rightarrow|\downarrow, 0\rangle$. In the third step, another individual photon is generated via the reverse LZ sweep $|\downarrow, 0\rangle \rightarrow|\uparrow, 1\rangle$. Fourth and finally, a further photon decay completes the cycle.

This scheme for repeated photon generation via Landau-Zener cycles makes use of two advantageous properties of circuit QED: first, the artificial atom is fixed at an antinode of the cavity, so that the atom-cavity coupling remains at a constant and high value. Second, qubits are highly tunable so that LZ sweeps can be made from minus to plus an "atomic" frequency, and back. The outlined scheme presents an alternative to the proposals for single-photon generation put forward in Refs. $[22,23]$. The main advantage of the present scheme is its robustness against parameter variations when operating in the regime $\gamma \ll \hbar \Omega$.

Experimental realisation. - In practice, the cavity frequency $\Omega$ and the qubit-oscillator coupling $\gamma$ are determined by the design of the setup, while the Josephson energy can be switched at a controllable velocity $v$ - ideally from $E_{\mathrm{J}}=-\infty$ to $E_{\mathrm{J}}=\infty$. In reality, however, $E_{\mathrm{J}}$ is bounded by $E_{\mathrm{J}, \max }$ which is determined by the critical current. The condition $E_{\mathrm{J}, \max }>\hbar \Omega$ is required so that the qubit comes into resonance with the oscillator sometime 


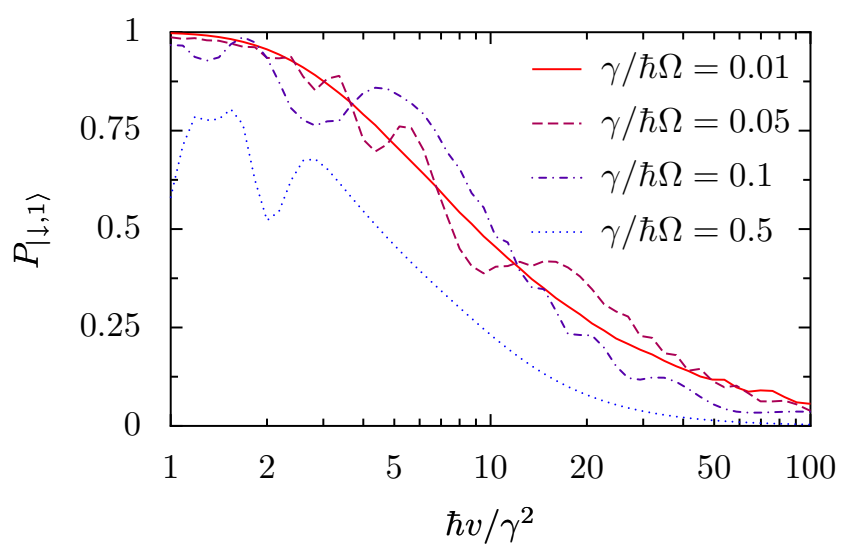

Fig. 3 - Probability of single-photon generation $P_{|\downarrow, 1\rangle}$ as a function of $\hbar v / \gamma^{2}$, for LZ sweeps within the finite time interval $[-T, T]$ with $T>T_{\min }$ chosen such that $v T=3 \hbar \Omega / 2$. The initial state is $|\uparrow, 0\rangle$. Shown probabilities are averaged within the time interval $\frac{29}{20} \hbar \Omega / v$ and $\frac{3}{2} \hbar \Omega / v$, whereby the small and fast oscillations that are typical for the tail of a LZ transition are averaged out.

during the sweep. Moreover, inverting the flux through the superconducting loop requires a finite time $2 T_{\min }$, so that $v$ cannot exceed $v_{\max }=E_{\mathrm{J}, \max } / 2 T_{\min }$. In order to study under which conditions the finite initial and final times can be replaced by $\pm \infty$, we have numerically integrated the Schrödinger equation in a finite time interval $[-T, T]$. Results are presented in Fig. 3. The first three curves in Fig. 3 correspond to the experimentally relevant regime where $\gamma \ll \hbar \Omega$. Here the essential dynamics is a LZ transition from $|\uparrow, 0\rangle$ to $|\downarrow, 1\rangle$ around the time $\hbar \Omega / v$. Especially for the smallest coupling constants shown, the probability for singlephoton generation practically equals $P_{\uparrow \rightarrow \downarrow}(\infty)$ given by Eq. (7). Thus we find that finite-time effects do not play a role as long as $\gamma \ll \hbar \Omega$. Our predicted transition probabilities based on analytical results for infinite propagation time are therefore useful to describe the finitetime LZ sweeps. Figure 3 also illustrates that the probability for single-photon production is highest in the adiabatic regime $\hbar v / \gamma^{2} \ll 1$. Here the typical duration of a LZ transition is $2 \gamma / v[16,24]$. So in the regime of interest, the sought condition for a "practically infinite time interval" is $v T=E_{\mathrm{J}, \max }>\hbar \Omega+2 \gamma$. For the unrealistically large qubit-oscillator coupling $\gamma / \hbar \Omega=0.5$, reliable single-photon generation is less probable. This is so because (i) the LZ transition is incomplete within $[-T, T]$; (ii) more than two oscillator levels take part in the dynamics and more than one photon can be generated, as depicted in Fig. 2 and (iii) the approximation of the instantaneous ground state at $t=-T$ by $|\uparrow, 0\rangle$ is less accurate.

For the setup of Refs. [2,3], a typical cavity frequency is $\Omega=2 \pi \times 10^{9} \mathrm{~Hz}$. The sign of the initial Josephson energy $E_{\mathrm{J}, \max }=2 \pi \hbar \times 10^{10} \mathrm{~Hz}$ can be inverted within $T=1 \mu$ s so that $v_{\max }=2 \pi \hbar \times 10^{16} \mathrm{~s}^{-2}$. For the qubit-oscillator coupling strength we assume $\gamma / 2 \pi \hbar=3 \times 10^{6} \mathrm{~Hz}$. Notice that $\gamma \ll \hbar \Omega$, so that generation of more than one photon is negligible. Then, by choosing $v=0.05 v_{\max }$, one finds $\left|c_{1}\right|^{2}>1-10^{-7}$ and a superposition with equal weights for which $P_{\uparrow \rightarrow \uparrow}(\infty)=\left|c_{1}\right|^{2} P_{\uparrow \rightarrow \downarrow}(\infty)=0.5$ is obtained. By choosing $v=v_{\text {max }}$ instead, a nonadiabatic transition occurs with the probability $P_{\uparrow \rightarrow \uparrow}(\infty) \approx 0.97$, while the remaining probability again corresponds to single-photon generation. As another extreme case, for the much slower sweep velocity $v=0.01 v_{\max }$, one is in the adiabatic limit in which a single photon is created with a probability of $\left|c_{1}\right|^{2} P_{\uparrow \rightarrow \downarrow}(\infty)=0.97$. Thus for the final state, one can obtain any desired superposition of the states $|\uparrow, 0\rangle$ and $|\downarrow, 1\rangle$ by choosing a proper value of $v$. 
In summary, we have shown that the coupling of a qubit to a circuit cavity mode induces Landau-Zener transitions upon variation of the penetrating flux. For an oscillator initially in its ground state, we derived an exact closed expression for the transition probability which possesses an appealing form: it only depends on the interaction strength between the qubit and the oscillator and, in particular, it is independent of the actual oscillator frequency $\Omega$. Thus, the LZ dynamics can be manipulated via the velocity at which the qubit levels cross. For a circuit QED setup, we found that both nonadiabatic and adiabatic sweeps are feasible and can be exploited for quantum state preparation. Repeated adiabatically slow Landau-Zener sweeps allow the controlled and robust creation of single photons.

We thank A. Wallraff for helpful discussions. This work has been supported by the Freistaat Bayern via the "Quantum Information Highway A8", the DFG through SFB 631, and a Grant-in-Aid for Scientific Research of Priority Area from the Ministry of Education, Sciences, Sports, Culture and Technology of Japan (No. 14077216).

\section{REFERENCES}

[1] Nakamura Y., Pashkin Y. A., and Tsai J. S., Nature (London), 398 (1999) 786.

[2] Wallraff A., Schuster D. I., Blais A., Frunzio L., Huang R.-S., Majer J., Kumar S., Girvin S. M., and Schoelkopf R. J., Nature (London), 431 (2004) 162.

[3] Blais A., Huang R.-S., Wallraff A., Girvin S. M., and Schoelkopf R. J., Phys. Rev. A, 69 (2004) 062320.

[4] Chiorescu I., Bertet P., Semba K., Nakamura Y., Harmans C. J. P. M., and MooiJ J. E., Nature (London), 431 (2004) 159.

[5] Landau L. D., Phys. Z. Sowjetunion, 2 (1932) 46.

[6] Zener C., Proc. R. Soc. London, Ser. A, 137 (1932) 696.

[7] Stueckelberg E. C. G., Helv. Phys. Acta, 5 (1932) 369.

[8] Saito K. and Kayanuma Y., Phys. Rev. B, 70 (2004) 201304(R).

[9] Hicke C., Santos L. F., and Dykman M. I., Phys. Rev. A, 73 (2006) 012342.

[10] Ankerhold J. and Grabert H., Phys. Rev. Lett., 91 (2003) 016803.

[11] Izmalkov A. et al., Europhys. Lett., 65 (2004) 844.

[12] Ithier G., Collin E., Joyez P., Vion D., Esteve D., Ankerhold J., and Grabert H., Phys. Rev. Lett., 94 (2005) 057004.

[13] Oliver W. D., Yu Y., Lee J. C., Bergaren K. K., Levitov L. S., and Orlando T. P., Science, 310 (2005) 1653.

[14] Sillanpä̈̈ M., Lehtinen T., Paila A., Makhlin Y., and Hakonen P., Phys. Rev. Lett., 96 (2006) 187002.

[15] Wernsdorfer W., Bhaduri S., Vinslava A., and Christou G., Phys. Rev. B, 72 (2005) 214429.

[16] Wubs M., Saito K., Kohler S., Kayanuma Y., and Hänggi P., New J. Phys., 7 (2005) 218.

[17] ViOn D. et al., Science, 296 (2002) 886.

[18] Kayanuma Y., J. Phys. Soc. Jpn., 53 (1984) 108.

[19] Grifoni M. and Hänggi P., Phys. Rep., 304 (1998) 229.

[20] Sinitsyn N. A., J. Phys. A: Math. Gen., 37 (2004) 10691.

[21] Volkov M. V. and Ostrovsky V. N., J. Phys. B: At. Mol. Opt. Phys., 38 (2005) 907.

[22] Liu Y.-X., Wei L. F., and Nori F., Europhys. Lett., 67 (2004) 941.

[23] Mariantoni M., Storcz M. J., Wilhelm F. K., Oliver W. D., Emmert A., Marx A., Gross R., Christ H., and Solano E., (2006). cond-mat/0509737

[24] Vitanov N. V., Phys. Rev. A, 59 (1999) 988. 